\title{
Design Technique and modification of Power line filter using passive lumped components
}

\author{
Shashwatee Paul ${ }^{1}$, Padmadhar Garg ${ }^{2}$, Ankit Barasiya ${ }^{3}$ \\ ${ }^{1,}$ (ECE, Gitam University, India) \\ 2. (EEE, NIT Jaipur, India) \\ ${ }^{3}(U T D$ RGPV, Bhopal, India)
}

\begin{abstract}
A unique design technique of power line filter is being proposed. It is based on the conventional technique of composite low pass filter. The equivalent circuit of the composite low pass filter is being presented in the two different modes by considering the modal propagation of noise. The consideration of composite LPF is taken into account in the design technique of power line filter. In order to accurately predict the insertion loss of the filter the effects of non-ideal behaviour of the passive lumped components are considered. It is based on the parasitic effect of the passive lumped elements. The filter is then characterized using S-parameters. The response of the filter's circuits is simulated using advanced design software (ADS).
\end{abstract}

Keywords: Conducted emission, Composite filter, electromagnetic compatibility, lumped elements, and power line filter.

\section{Introduction}

Switching and modulation techniques are used for efficient use of electrical power. Widely used Switched mode power supplies (SMPS) for powering today's electronics loads is a most common example. Unfortunately, all the power control techniques deliberately distort sinusoidal wave form of power frequency and generate unwanted interfering signals. Obviously, they all are often cited as one of the main source of conducted emission (CE). Usually the power supplies which are using controlling techniques cannot comply with the strict electromagnetic compatibility (EMC) regulations. In order to meet the limit set for conducted EMI, power supplies require a filter at its input. Conducted EMI generated through particular equipment gets coupled into other equipments mostly through power line cables. CE can be controlled by using filters and increasing line impedance. Conducted EMI has two components: The common mode (CM) interference and differential mode (DM) interference. The coupling mechanisms of these interferences are different [4]. The difference in the direction of propagation of these coupling currents along the phase and neutral line, leads to differential mode $\left(I_{d m}\right)$ current and common mode $\left(I_{c m}\right)$ current. Therefore design of EMI filters demand decoupling of these two modes [2]. This paper attempts to describe a design technique for Power Line filter using classical design technique.

\subsection{Characterization of common mode \& differential mode currents}

The currents flowing through the phase and neutral conductors are depicted as $\left(I_{p}\right)$ and $\left(I_{n}\right)$. These currents can be decomposed into two auxiliary currents, which are referred to as the common mode current $\left(I_{c m}\right)$ and differential mode current $\left(I_{d m}\right)$ [5]. The propagation of interferences through phase, neutral and ground conductor is shown in the fig (1) [6].
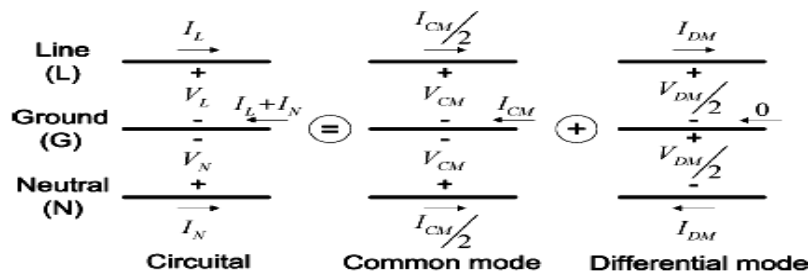

Fig1: Relationship between circuital and modal voltages and currents.

The CM and DM currents separately can be determined from the conducted emission measurement data:

$$
\begin{array}{ll}
I_{p}=I_{c m}+I_{d m} & I_{n}=I_{c m}-I_{d m} \\
I_{c m}=\frac{I_{p}+I_{n}}{2} & I_{d m}=\frac{I_{p}-I_{n}}{2}
\end{array}
$$

Input impedance of measuring instruments is mostly $50 \Omega$. Therefore, the CM and DM Voltage can be defined as:

$$
V_{d m}=50 I_{d m}=50\left(\frac{I_{p}-I_{n}}{2}\right)=\frac{V_{p}-V_{n}}{2}
$$




$$
V_{c m}=50 I_{c m}=50\left(\frac{I_{p}+I_{n}}{2}\right)=\frac{V_{p}+V_{n}}{2}
$$

The equivalent circuit for the conducted emission measurement setup is shown in the fig2 [5]

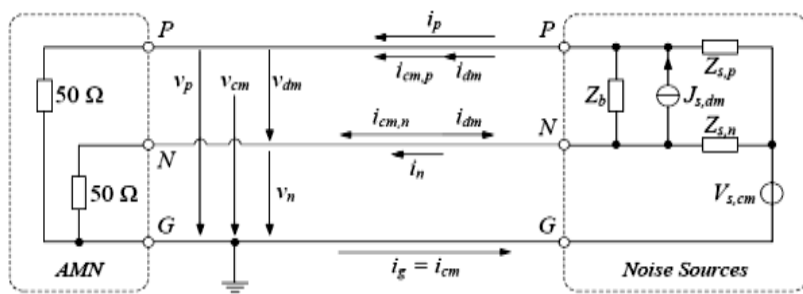

Fig2: Equivalent circuit of the conducted emissions measurement.

\subsection{Conducted emission measurement}

An example of a measurement setup for conducted emission tests according to CISPR 16-2-1is carried out at the centre of SAMEER Kolkata as shown in the above figure (3). The EUT is supplied by the power source through an artificial mains network (AMN).It consists of two line impedance stabilization networks (LISNs), one for phase line and the other for neutral line. Measurement is carried out in frequency domain the graphical display of the conducted emission is shown in the EMI Receiver.

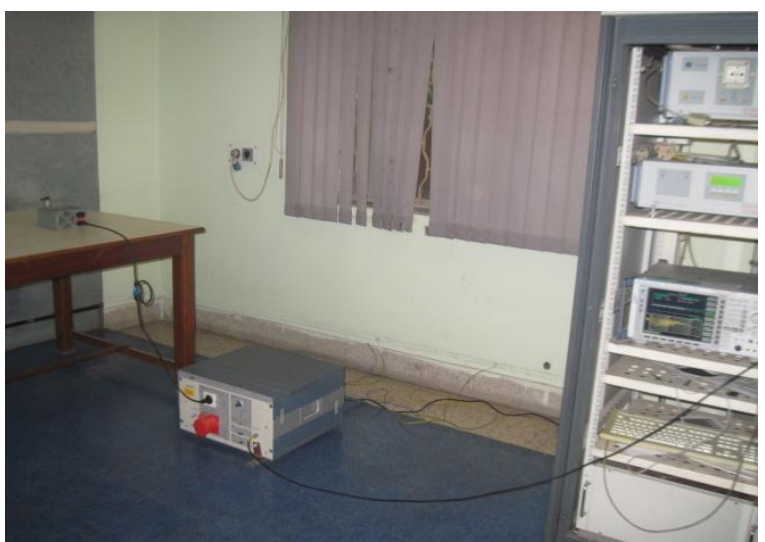

Fig 3: Measurement set up at SAMEER Kolkata

The measurement is carried with Line impedance stabilising network (LISN) and the the conducted emission test is viewed in the EMI Test receiver. The measurement data of the test Receiver is shown in the fig (4). From the data of conducted emission test, it can be viewed that the peak level of emission exists at $80 \mathrm{~dB} \mu \mathrm{V}$. But according to the standard specification, the limitation of the emission is 40dB.Therefore according to the definition of Insertion loss, the filter must be designed in such a manner, that the attenuation of the power line filter must be more than $40 \mathrm{~dB}$.

\subsection{Conducted noise separation}
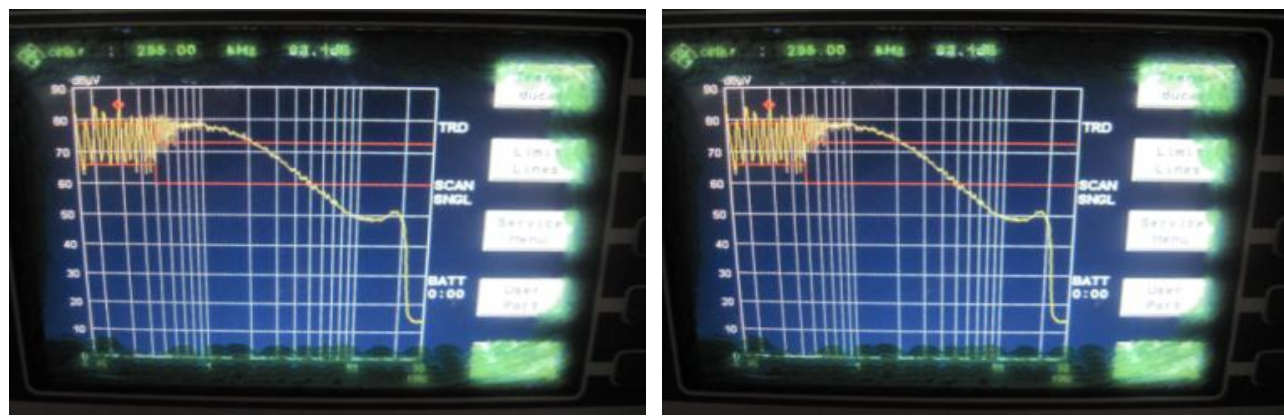

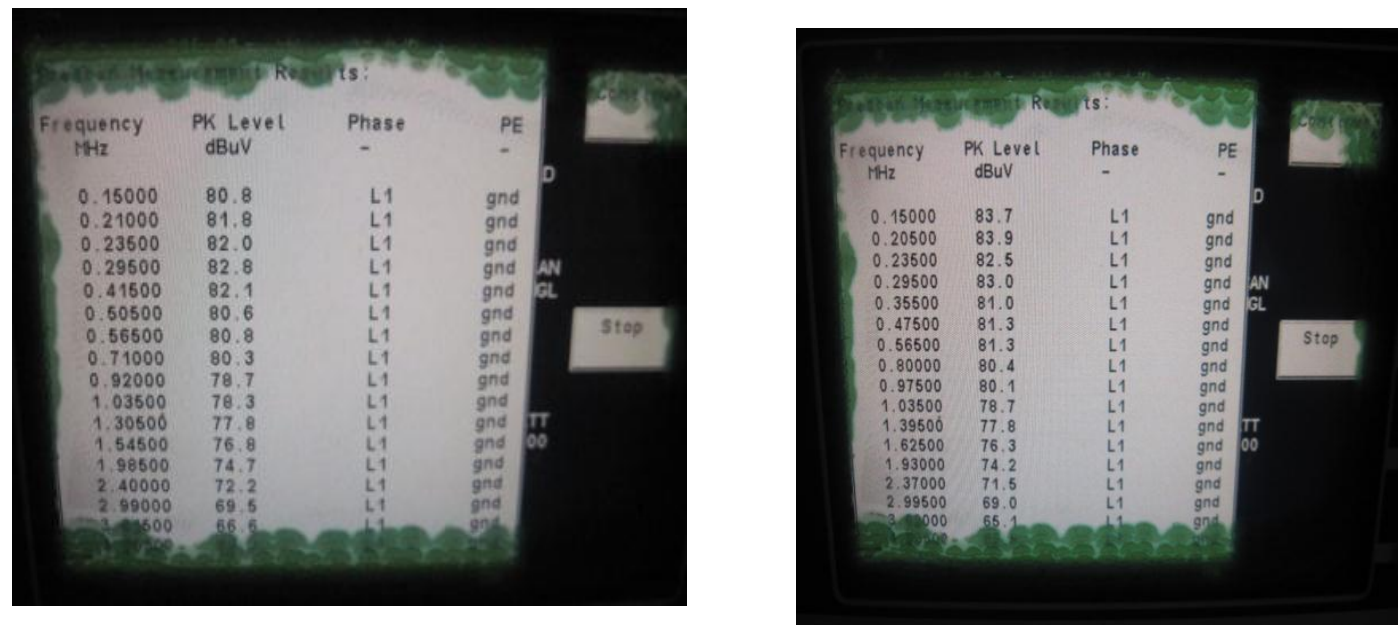

Fig 4: EMI Test Receiver data

The components of a power filter attenuate CM and DM disturbances differently. For this reason, it is impossible to design or select an EMI filter without knowing the level of the CM and DM noise. In this from the measurement test data the CM and DM noise voltages can be solved by taking the sum of $I_{p}$ and $I_{n}$. The CM and DM voltages can deduced from the voltage drop of the corresponding currents across the $50 \Omega$ resistor.

$$
v_{c m}=\frac{v_{p}+v_{n}}{2}=\frac{50 \Omega}{2}\left(i_{p}+i_{n}\right)=25 \Omega . i_{c m}
$$

which can be interpreted as if the CM current $i_{c m}$ sees two $50 \Omega$ resistances in parallel, i.e. $25 \Omega$, and this is independent of how $i_{c m}$ splits between the phase and the neutral.

But the DM voltage is defined in a different manner accordingly [5]

$$
v_{d m}=v_{p}-v_{n}
$$

When the noise currents are terminated by two $50 \Omega$ impedance, as in the conducted emissions tests, the relationship between the DM voltage and current is:

$$
v_{d m}=v_{p}-v_{n}=50 \Omega \cdot i_{p}-50 \Omega . i_{n}=2.5 \Omega \frac{i_{p}-i_{n}}{2} 100 \Omega . i_{d m}
$$

Therefore the DM voltage is the voltage drop over two $50 \Omega$ resistances in series, and the DM termination impedance is $100 \Omega$.

\section{Filter Topology Of Composite Low Pass Filter}

In this paper, we present a design methodology to realize low pass filter using a unique combination of constant-k, m-derived section. Constant-k section gives sharp cut-off while m-derived section provides the impedance matching at input and output sides of the LPF [3]. The m-derived T-section was designed so that its image impedance was identical to that of the constant-k section. The m-type filter section is a big improvement with suitable choice of the parameter $\mathrm{m}$. It has a further advantage in that there is a rapid transition from the cutoff frequency of the pass band to a pole of attenuation just inside the stop band. Cascading both constant-k and m-derived section gives a composite LPF, which gives both sharp cut off and good impedance matching[3].This type of LPF is called composite filter, which is designed separately for the common mode filter in the frequency range of $150 \mathrm{khz}$ to $1 \mathrm{Mhz}$ and the differential mode filter in the frequency range of $1 \mathrm{Mhz}$ to $30 \mathrm{Mhz}$.

Constant-k Section: The value of Capacitance and Inductance is determined [3] as

$$
L=\frac{Z_{o}}{\pi f_{c}} \quad C=\frac{1}{\pi f_{c} z_{o}}
$$

M-derived Section: The cut-off frequency $\left(f_{c}\right)$ and the infinite attenuation occurred at frequency $\left(f_{\infty}\right)$ is defined as

$$
f_{\infty}=\frac{f_{c}}{\sqrt{1-m^{2}}} \quad f_{c}=\frac{1}{\pi \sqrt{L C}}
$$

The independent element values of m-derived LPF is obtained as

$$
L^{\prime}=\frac{m l}{2} \quad C^{\prime}=m c L^{\prime \prime}=\frac{1-m^{2}}{4 m}
$$

Matching section: This Circuit can be further modified using the Matching section i.e. bisected- $\pi$ section of the M-derived section that are implemented as a terminating Half Section. The composite LPF is transformed into Common mode 
and Differential mode Power line section. The cut off frequency for the differential mode is $f_{c}: 50 \mathrm{kHz}$ and the infinite attenuation pole is $f_{\infty}: 1 \mathrm{Mh}$.

The lumped elements of the terminating half sections at the input and output port of the low pass filter can be determined as

$$
L^{\prime}=\frac{m l}{2} \quad C^{\prime}=\frac{m c}{2} L^{\prime \prime}=\frac{1-m^{2}}{2 m}
$$

\subsection{Equivalent circuit of Differential mode Filter}

The composite low pass filter is designed for the differential mode filter with the cut off frequency $f_{c}$ : $50 \mathrm{kHz}$ and infinite attenuation pole $f_{\infty}: 1 \mathrm{MHz}$. It consists of a constant-k filter section, m-derived section \& matching section at the input and output. The direction of propagation of DM current is considered while designing the circuit.

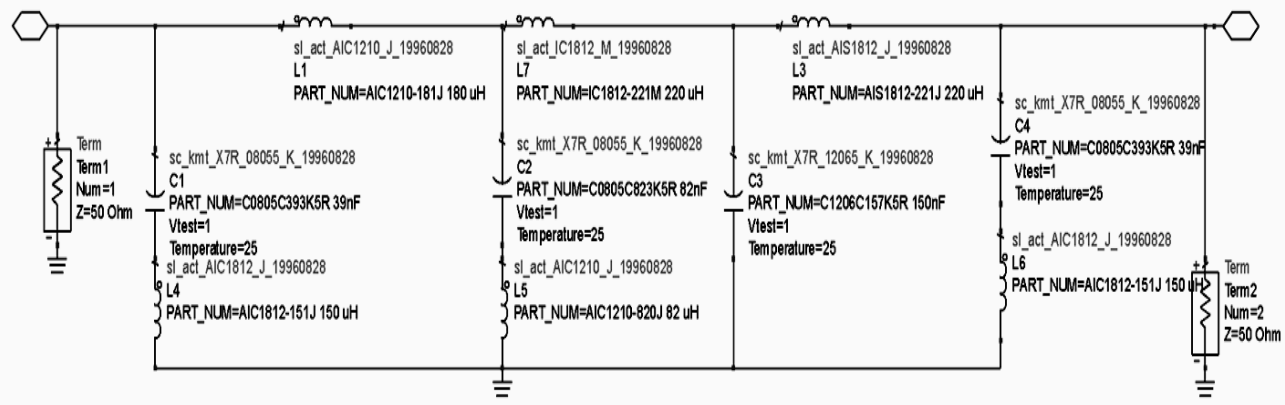

Fig5: Circuit of lumped element DM composite filter.

Equivalent circuit of Common mode Filter

In this circuit, the series inductors of DM section are connected in series with the circuit of CM filter. It is based on the propagation of CM current from source to load. The design is carried out at a cut off frequency $\left(f_{c}\right): 333$ $\mathrm{kHz}$ and maximum attenuation pole $\left(f_{\infty}\right): 30 \mathrm{MHz}$.

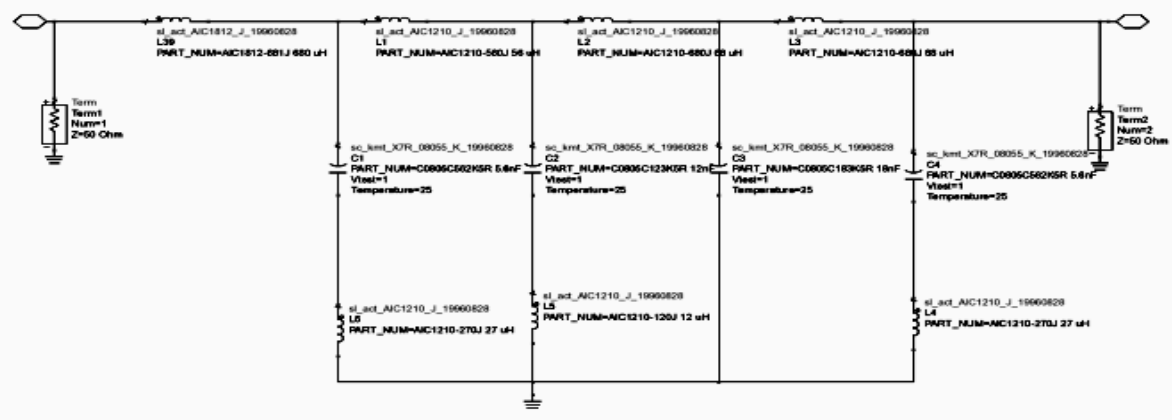

Fig 6: Schematic diagram of the modified circuit of CM Filter.

\subsection{Response of DM and CM filter circuit}

The differential mode \& common mode circuits are simulated in ADS based on S-parameter as shown in the figure.
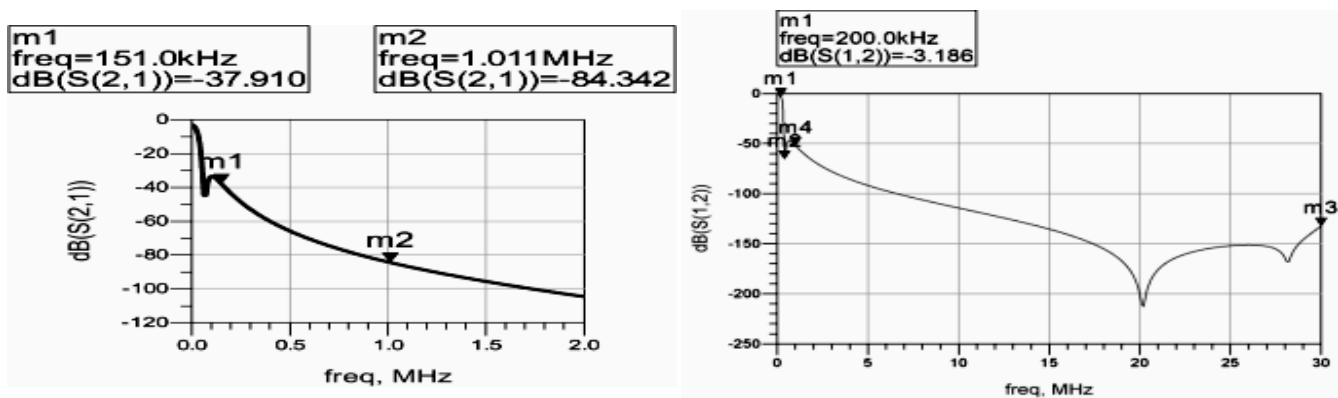

Fig7: Simulation result for DM \& CM composite filter.

The simulation result of the DM filter has an attenuation of $84 \mathrm{~dB}$ at the $1 \mathrm{MHz}$ frequency. While the $\mathrm{CM}$ filter shows an attenuation of $125 \mathrm{~dB}$ at the frequency of $30 \mathrm{MHz}$.

\subsection{Insertion loss of the filter}


The IL is defined as the ratio of the voltages appearing across the lines immediately beyond the point of insertion, before and after the insertion of the filter [8] The oldest book definition defines the IL as the insertion ration IR in $\mathrm{dB}$.

$$
\begin{aligned}
& \mathrm{IR}=\frac{V_{20}}{V_{2}}=\frac{I_{20}}{I_{2}} \\
& \mathrm{IL}=20 . \log \mathrm{IR}) \\
& \mathrm{IL}=20 . \log \left(\frac{I_{20}}{I_{2}}\right)=20 . \log \left(\frac{V_{20}}{V_{2}}\right)
\end{aligned}
$$

CISPR 17 specifies that in the standard IL measurements the input and output of the filter must be terminated in equal and fixed resistance - normally $50 \Omega$ to $75 \Omega$ [9]. In this the $50 \Omega$ fixed resistances are taken as source and load impedance.

\section{Role of parasitic effects of passive elements}

Capacitor: The mathematical models that yield considerable insight into the non ideal behaviour of components have been used. In the conducted emission range $(150 \mathrm{kHz}-30 \mathrm{MHz})$, the behaviour of these elements is far from the ideal. The model of the equivalent circuit of Capacitor consists of a series combination of series resistor $\left(R_{S}\right)$, lead inductor $\left(L_{\text {lead }}\right)$ and capacitor. The model of equivalent circuit of capacitor is as shown in fig (8). The lead inductance of the capacitor can be determined from its self resonant frequency (SRF). The impedance of the capacitor is given by:

$$
Z_{c}=R_{s}+j w l+\frac{1}{j w C}
$$

For low-frequency, a capacitor appears as high impedance, but for HF signals, such as noise currents in the 0.15 to $30 \mathrm{Mhz}$ range, it is low impedance. Therefore, capacitors are connected in parallel with the noise source. Non ideal behaviour of passive components through Simulation results: The non ideal response of the filter can be noticed from the frequency characteristic graph of the Common mode filter at higher frequency. It shows a ripple formation in the frequency response of the graph. This non ideal behaviour of the filter characteristic is due to the effect of parasitic components of the lumped elements (capacitor \& inductor). The non ideal response of the individual passive components was simulated as shown in the fig (9).

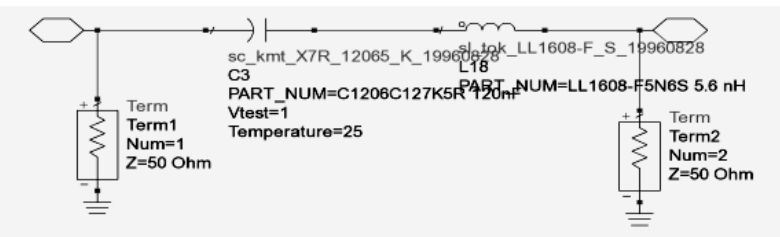

Fig8: Schematic diagram of the Equivalent circuit of Capacitor
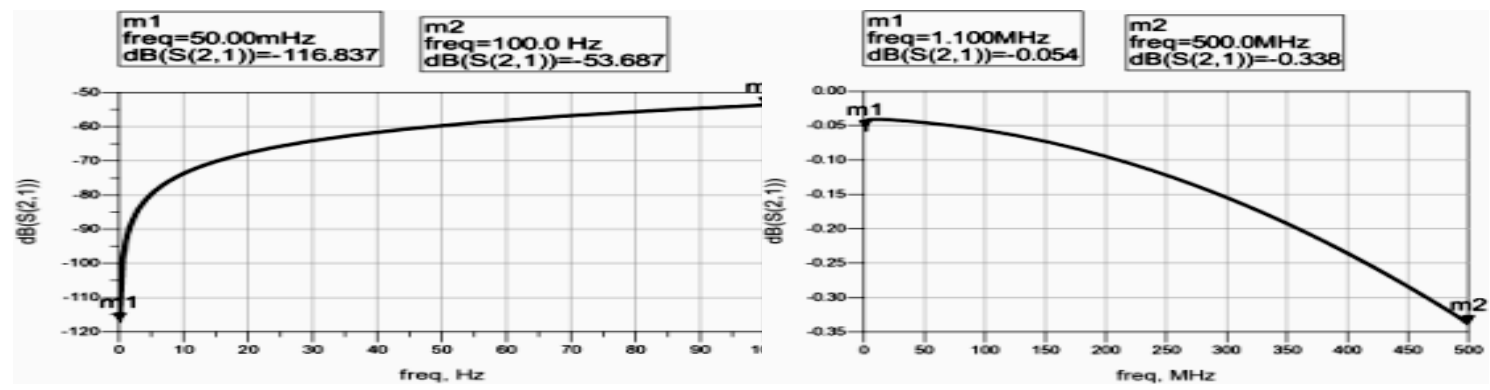

Fig9: Simulated Non-ideal response of the Capacitor with parasitic components

Inductor: The equivalent circuit of an inductor and the non ideal behaviour the inductor can be represented as in [1].The mathematical models that yield considerable insight into the non ideal behaviour of components have been used. In the conducted emission range $(150 \mathrm{kHz}-30 \mathrm{MHz})$, the behaviour is far from the ideal characteristic. The model of the equivalent circuit of Inductor consists of a series combination of parasitic resistor $\left(R_{\text {par }}\right)$, and a parasitic shunt capacitor $\left(C_{\text {par }}\right)$ as shown in (fig 10). The frequency response of the 
inductor shows that the inductor is dominant unto a certain frequency and after that, the parasitic capacitance become more dominant. The impedance of the inductor is given by:

$$
Z_{l}=\frac{R_{p a r}+j w l}{1-w^{2} j w L C+j w R_{p a r} C}
$$

The non ideal response of the filter can be noticed from the frequency characteristic graph of the Common mode filter at higher frequency. It shows a ripple formation in the frequency response of the graph. This non ideal behaviour of the filter characteristic is due to the effect of parasitic components of the lumped elements (capacitor \& inductor). The non ideal response of the individual passive components was simulated as shown in the fig (10).
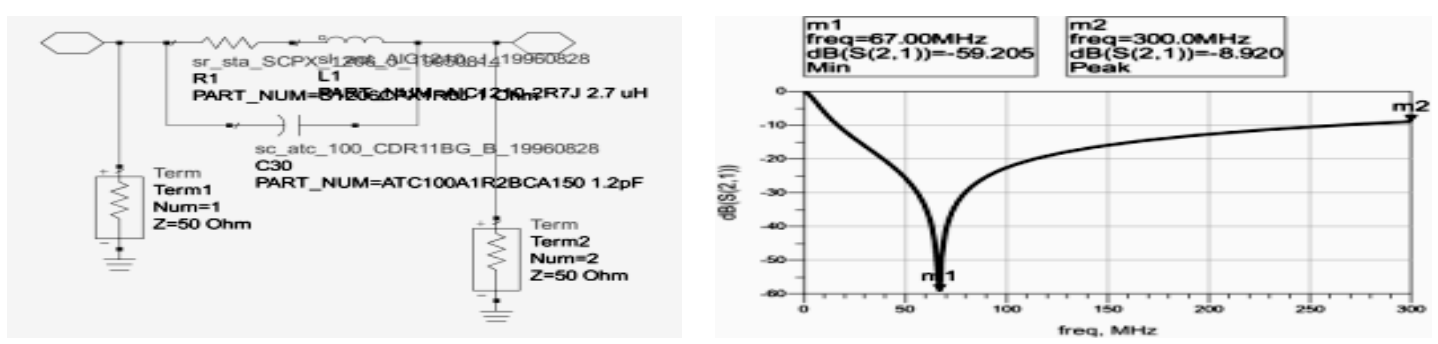

Fig10: Equivalent circuit of an Inductor and its simulated IL response

Each of the passive elements can have good high frequency characteristics due to the presence of the ever present parasitic. As a result of their presence, any suppression component resonates at some frequency, namely the self-resonant frequency (SRF), given by

$$
f_{S R F}=\frac{1}{2 \pi \sqrt{L C}}
$$

The concentration is mainly on their role in suppression of conducted emissions and on their non ideal behaviour [1]. Over a certain range of frequency, the total current of interference is the resultant of the dominant component current.

\section{Equivalent circuit of the filter}

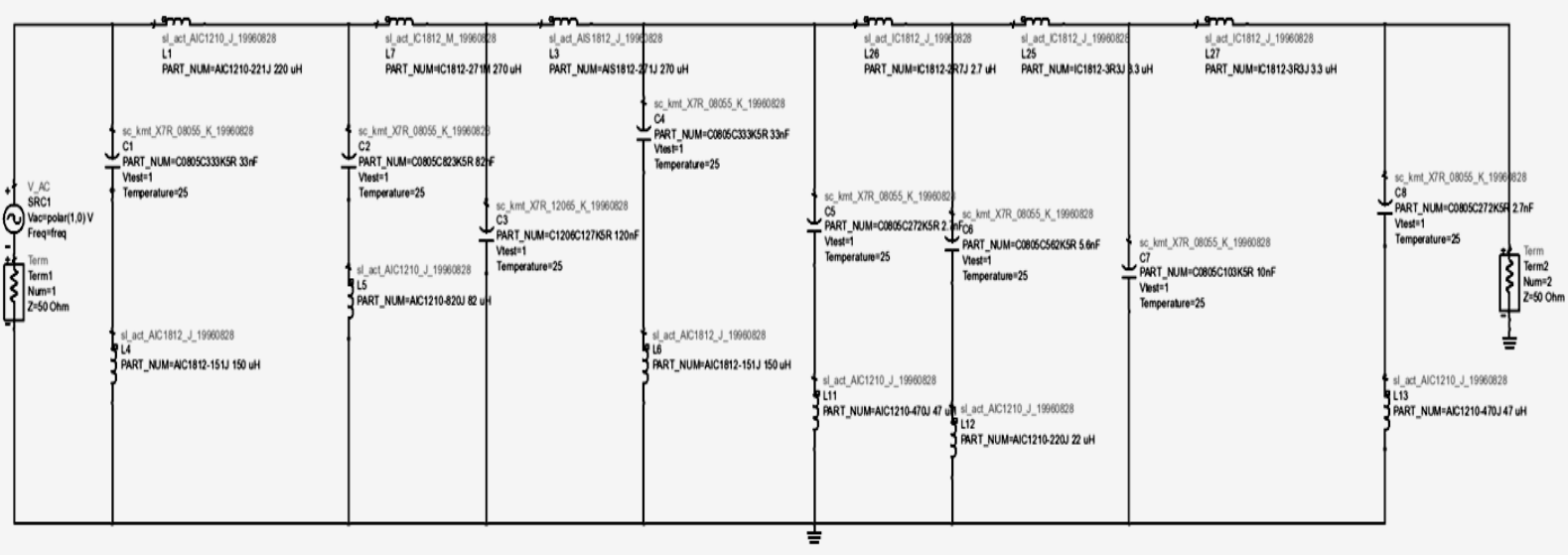

Fig11: ADS schematic of DM filter

The ADS schematic circuit of the complete filter design is shown in the above figure. The equivalent circuit of the power line filter is based on the cascaded connection of differential mode (DM) filter section and the common mode (CM) filter section as shown in the above (fig11). The source end of the filter circuit is connected to DM section and the load terminal is connected to the CM section. The frequency response of the filter is shown in the (fig 12).

In order to get the desired IL and to meet the frequency characteristics of practical filter circuit, the filter circuit is further modified as shown in (fig 12). The circuit is now a balanced network. The frequency response is enhanced w.r.t the original filter circuit. 


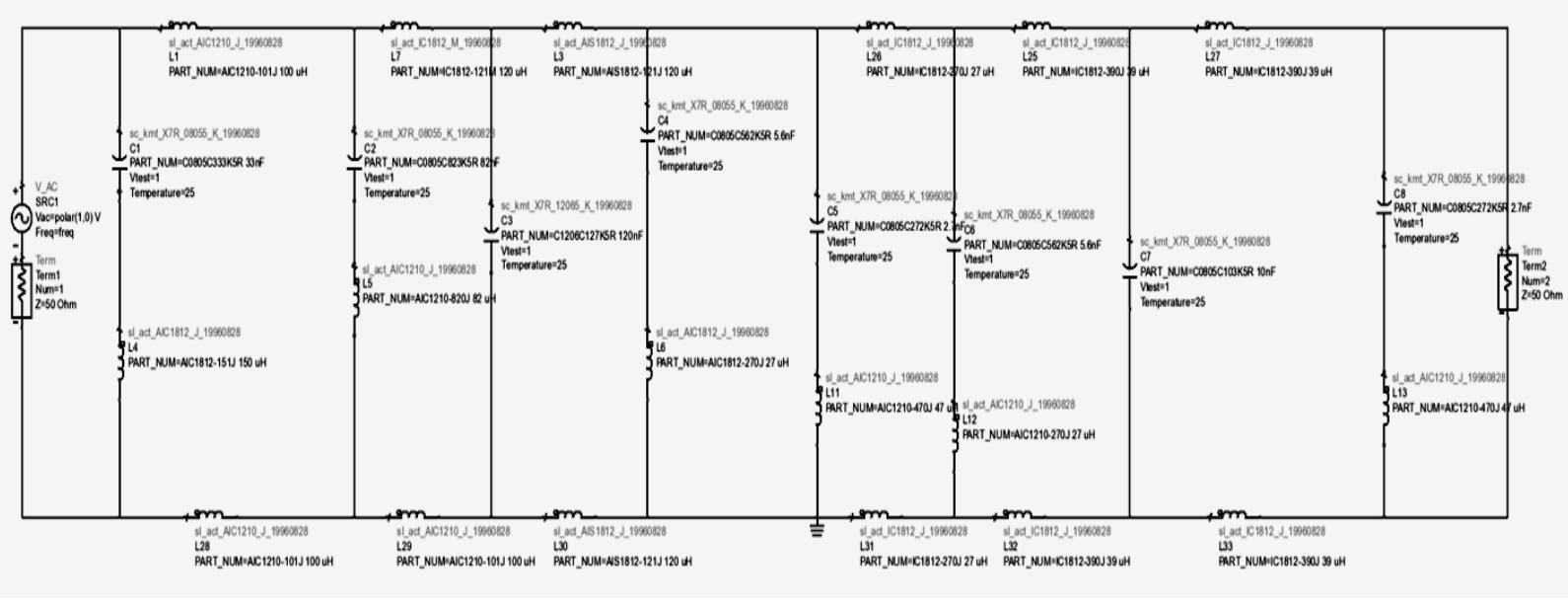

Fig12: ADS schematic of CM filter

\section{Result}

Simulation result of the modified filter circuit Simulation result for DM filter

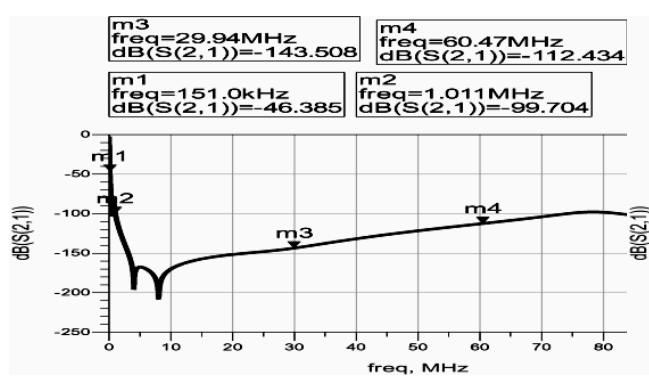

Fig13: Simulation result of the modified DM filter.

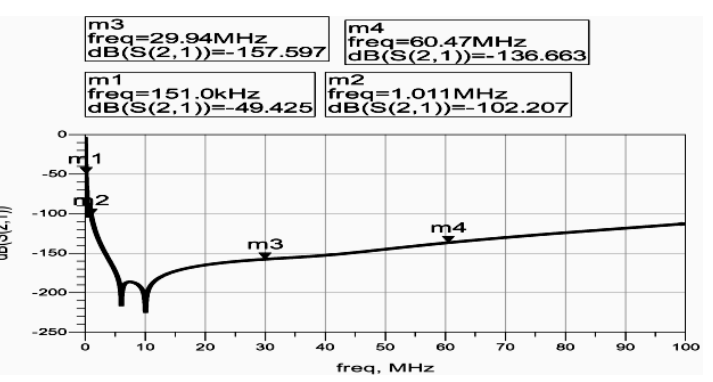

Fig14: Simulation result of the modified CM filter

\section{Conclusion}

- From the simulation result of the filter circuit it is noticed that, design of power line filter using constant-k and m-derived composite low pass filter gives a better frequency response.

- The design of power line filter with composite LPF is simple and hence it avoids any complicacy

- From the simulation result of the modified filter circuit, it is clear that the desired attenuation is achieved even beyond the cut off frequency.

- The performance of the filter response are enhanced when the parasitic effect of the lumped elements are considered in the modified circuit.

\section{Books:}

\section{Reference}

[1] Clayton R.Paul, Electromagnetic compatibility, J.Wiley\& Sons, Inc.

[2] V.Prasad kodali, Engineering Electromagnetic Compatibility, The institute of Electrical and Electronics Engineers, Inc, New York

[3] D.M. Pozer, Microwave Engineering, J.Wiley \& Sons, Inc.

\section{Journal Papers:}

[4] Mohit Kumar and Vivek Agarwal, Power line filters Design for Conducted EMI using Timi-domain measurements, IEEE transaction. On electromagnetic compatibility, Vol.48, NO.1, February 2006

[5] Konstantin Kostov, Design and Characterization of Single-Phase Power Filters, Helsinki University of Technology.

[6] Albert Miquel Sanchez, Joan Ramon Regue, Miquel Ribo, Pablo Rodriguez-Cepeda and Francisco Javier Pajares, "A Modal Model of Common-Mode Chokes for Conducted Interference Prediction", IEEE transaction, On electromagnetic compatibility, Vol.52, NO.3. August 2010

[8] CISPR17, Methods of Measurement of the Suppression Characteristics of Passive Radio Interference Filters and Suppression Components, IES, Geneva, SWITZERLAND, 1981.

[9] W.C. Johnson: Transmission Lines and Network, McGraw-Hill Inc., 1950, p.361.

\section{Thesis:}

[7] Konstantin Kostov and JormaKyyra, “Insertion loss and Network parameters in the Analysis of Power Filters”, Nordic Workshop on Power and Industrial Electronics, Espoo, Finland, June 2008. 